\title{
Optically/Electrically (Symmetrically) Triggerable Bistable Multivibrator
}

M. K. Ravishankar and M. Satyam

\begin{abstract}
An optically and electrically, symmetrically triggerable hybrid bistable multivibrator giving both optical and electrical outputs is proposed, designed, and implemented using phototransistors, light emitting diodes, etc. The circuit is found to be working satisfactorily at medium frequency range.
\end{abstract}

\section{INTRODUCTION}

The need for optically controlled bistable, astable, and tristable devices is amply explained from the point of optical computing

Manuscript received November 15, 1995. This paper was recommended by Associate Editor H.-D. Chiang.

The authors are with the Department of Electrical and Communication Engineering, Indian Institute of Science, Bangalore-560 012, India.

Publisher Item Identifier S 1057-7122(96)04864-7. 
[1]-[4]. The importance of electrically triggerable bistable circuit for similar applications is very well known [5]. But for efficient and economical computing systems, circuits which can take care of the existing electrical signal domain and the futuristic optical signal domain are needed. They should have the flexibility of providing adaptability as well as time sharing for optical and electrical signals during computation. This necessitates the development of circuits which can operate on optical or electrical signals and provide both optical and electrical outputs. Having regard to such an idea, the basic sequential circuit used in computation, a Bistable multivibrator to satisfy the above requirements which we call hybrid Bistable multivibrator is proposed in this letter.

A conventional bistable multivibrator is a regenerative circuit essentially consisting of two cross coupled inverter stages made up of a transistor and a resistor having two stable states. The state of the circuit can be changed by applying appropriate ellectrical trigger pulse to the bases of the transistors. If one has to effect a transition by optical means, it is imperative to use phototransistors. Further to have optical outputs, the use of light emitting eleiments becomes necessary. Keeping these in view a Bistable multivibrator which is triggerable by symmetrical optical or electrical pulses and provide both optical and electrical output has been conceived and described here with relevant design and operational details.

\section{Design and Operation}

The circuit is developed by introducing suitable modifications to the conventional Bistable Multivibrator circuit to make it triggerable by symmetrical optical or electrical pulses and provide optical and electrical outputs. The circuit diagram is shown in Fig. 1(a).

It consists of two cross-coupled inverter circuits each of which is made up of one photo transistor with it's collector terrninal connected to a resistor and a light emitting diode to provide electrical and optical outputs, respectively. The light emitting diodes are located in space such that high optical intensity and high voltage corresponds to a logical ONE and vice versa for a logical ZERO(outputs).

The circuit parameters are arrived at using the optical and electrical characteristics of the Photo transistor, the light emitting diode and pursuing the design similar to that of a conventional Bistable multivibrator. It is found from the characteristics that a current of $10 \mathrm{~mA}$ flowing through the light emitting diode produces adequate optical intensity to induce sufficient current in the base of the phototransistor to generate a large current through the collector. Therefore a collector saturation current of $10 \mathrm{~mA}$ is chosen for the phototransistor which also flows through the light emitting diode in series with the collector and produces adequate optical intensity to drive the subsequent stage if any.

The values of other components required for achieving the stable state for the circuit are obtained as follows.

For a supply voltage of $5 \mathrm{~V}$, collector emitter saturation voltage of $0.8 \mathrm{~V}$, and a voltage drop of $2.1 \mathrm{~V}$ across light emitting diode of the conducting inverter, the value of collector load resistance $R_{c}$ to limit the collector saturation current to $10 \mathrm{~mA}$ works out to be $210 \Omega$.

The phototransistor in the conducting inverter stage requires a minimum base current of $0.44 \mu \mathrm{A}$ at a base to emitter voltage of $1.3 \mathrm{~V}$ to be in saturation. This current and voltage are provided by the current that flows through the light emitting diode connected in the collector circuit of the phototransistor pertaining to the non conducting inverter stage and resistors $R_{1}$ and $R_{2}$ connected to the base of the phototransistor belonging to the conducting inverter stage. This current has to be less than $30 \mu \mathrm{A}$ to keep the optical intensity from the light emitting diode in the non conducting inverter stage negligible. The voltage drop across the light emitting diode at this value of current is $1.7 \mathrm{~V}$. Using these data the value of $R_{1}$ and $R_{2}$

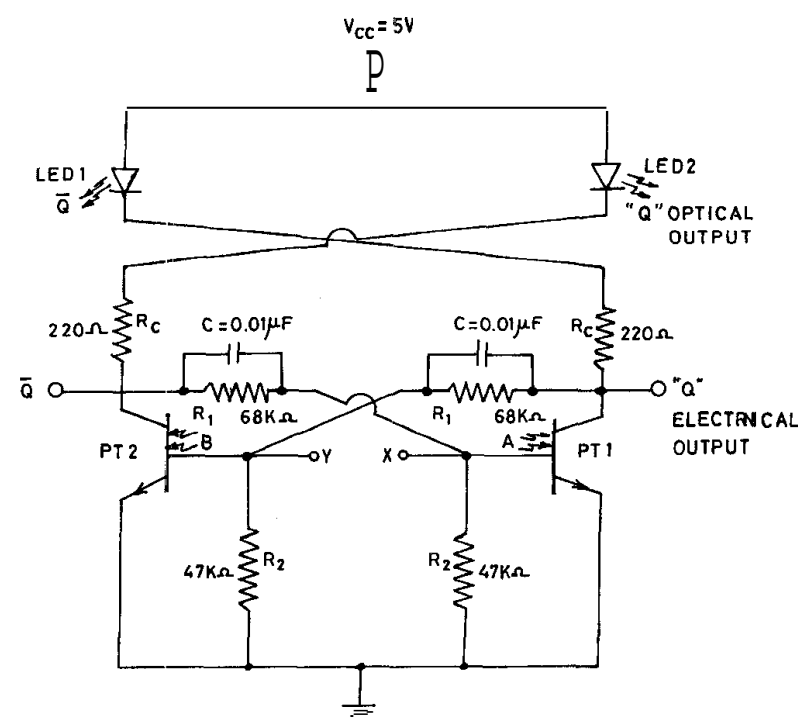

(a)

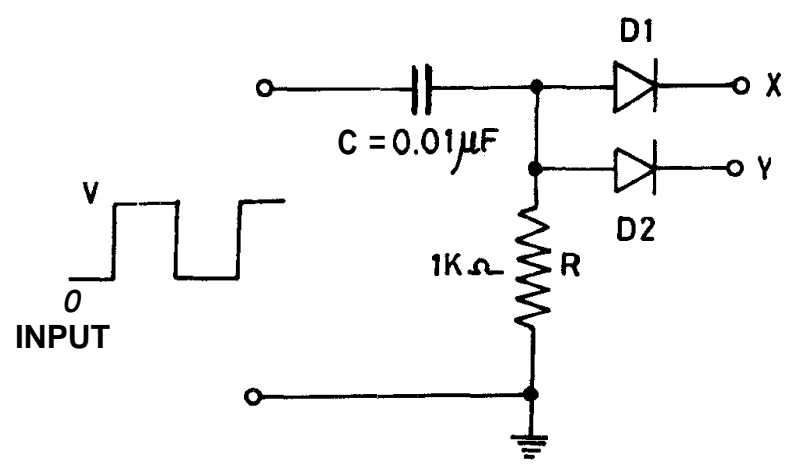

(b)

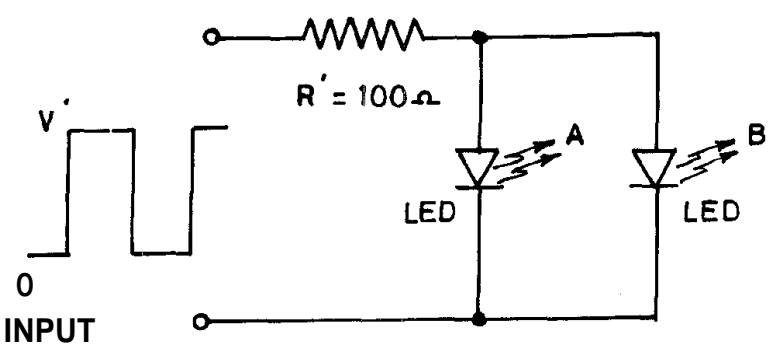

(c)

Fig. 1. (a) Schematic diagram of the Hybrid Bistable Multivibrator using Phototransistors L14F1 9220 and LED's, PT-Phototransistor. (b) Electrical triggering circuit. (c) Optical triggering circuit.

works out to be $68 \mathrm{KR}$ and $43.5 \mathrm{~K} \Omega$. The base to emitter voltage of the phototransistor in the nonconducting inverter stage is $0.3 \mathrm{~V}$ with these values of $R_{1}$ and $R_{2}$ and is much less than the base to emitter cut-in voltage of $1.15 \mathrm{~V}$. Thus the values of components obtained ensures that when one of the phototransistors is in saturation the other is in cut off. Thus a stable state is achieved for the circuit. For speedup of transition, commutating capacitors of value $0.01 \mu \mathrm{F}$ is used across $R_{1}$ which provides adequate compensation.

The symmetrical electrical triggering circuit consists of a resistor, capacitor and diode network connected to the bases of the photo- 


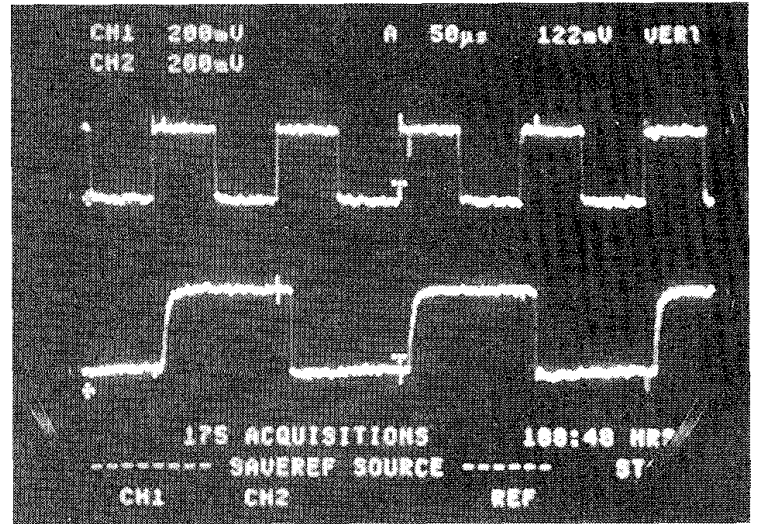

(a)

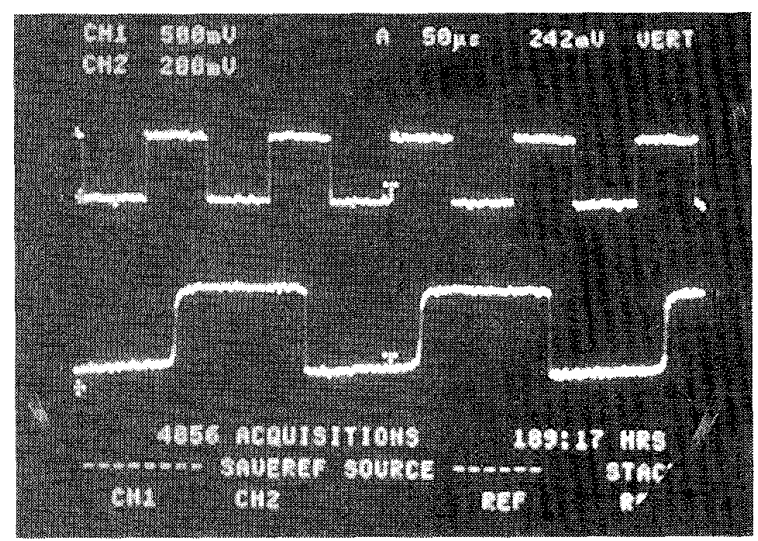

(b)

Fig. 2. Photographs depicting waveforms at the collector of one of the phototransistors with respect to input pulse: (a) Electrical triggering. (b) Optical triggering. Horizontal scale-Frequency. Vertical scale-Amplitude. $10: 1$.

transistors whereas focussing light intensity from two matched light emitting diodes onto the collector-base junctions of the phototransistors constituted the symmetrical optical triggering circuit as shown in Fig. 1(b) and (c). An electrical pulse applied at the input of any of these circuits forces conduction in the non conducting phototransistor instantaneously, which in turn drives the conducting phototransistor below cut off giving rise to a change in the state of the circuit.

In this circuit a logical ZERO is represented by a low voltage $(0.8$ V) and low light intensity output $(-30 \mathrm{dBm})$ whereas a logical ONE is represented by a high voltage $(3.1 \mathrm{~V})$ and high optical intensity $(-17 \mathrm{dBm})$ at the output.

\section{EXPERIMENT AND RESULTS}

The circuit is constructed using the components of standard specifications. The electrical and optical triggering is done using the circuits shown in Fig. 1(b) and (c). The source used is a Hewlett Packard Function Generator $8116 \mathrm{~A}$. The circuit has satisfactorily operated up to a frequency of $10 \mathrm{kHz}$. The electrical output at the collector of the phototransistor varies from 0.75 to $3.2 \mathrm{~V}$ and optical output from the light emitting diode varies from $-30 \mathrm{dBm}$ to $-17 \mathrm{dBm}$ for logical ZERO to logical ONE state. The minimum input pulse amplitude required is approximately 2 volts for electrical triggering and -17 $\mathrm{dBm}$ for optical triggering. The waveforms with the amplitude levels of input and output displayed on Tektronix 2432A Oscilloscope pertaining to the circuit for a square wave trigger under electrical and optical triggering are shown in Fig. 2(a) and (h).

The time delay between the input and output digital signals which indicates the speed with which the circuit can work satisfactorily is about $15 \mu \mathrm{s}$ and $10 \mu \mathrm{s}$ for optical triggering and electrical triggering respectively with the input pulse amplitudes shown in Fig. 2(a) and (b). This improves to about $7 \mu$ s maximum with higher input amplitudes for both optical and electrical triggering for the present circuit which is normal with the kind of phototransistors used [6]. The speed of operation of the circuit can be increased considerably by inducing a base bias current either by electrical or optical means and the use of high gain, high bandwidth heterojunction phototransistors [7], [8]. The use of differential output levels for the logical states instead of cut off and saturation levels wherein the storage charge effects are reduced can also enhance the speed. Further a reduction in the value of passive components can also bring in a improvement in speed when used with proper phototransistors and light emitting diodes.

The fan-out of the circuit gets enhanced by a higher collector current and a higher optical intensity output from the light emitting diodes connected to the collector of the phototransistor.

\section{CONCLUSION}

This letter describes a hybrid bistable multivibrator using phototransistors and light emitting diodes which is triggerable by symmetrical optical/electrical pulses and gives optical and electrical outputs. The Bistable multivibrator being a basic building block in sequential circuits, can form the basis for the growth of new branch of hybrid circuits usable in digital computation.

\section{REFERENCES}

[1] S. Noda, E. Yamada, T. Takayama, and A. Sasaki, "Light controlled optical bistable device by bistable laser diode and heterojunction phototransistors," Electron. Lett., vol. 26, no. 14, pp. 1069-1070, 1990.

[2] T. Chino, K. Matsuda, H. Adachi, and J. Shibata, "Novel optoelectronic RS-flipflop based on optically coupled inverters," Electron. Lett., vol. 28, no. 7, pp. 641-642, 1992.

[3] S. Noda, T. Katamata, K. Machori, K. Shibata, and A. Sasaki, "Optical fipflop and astable multivibrator functions by vertical and direct integration of heterojunction phototransistors and laser diodes," Opt. Rev., vol. 1, no. 2, pp. 213-216, 1994.

[4] S. Noda. Y. Kobayashi. K. Shibata, and A. Sasaki, "Optoelectronic integrated tristable device with optically controlled set and reset functions," IEEE. J. Quantum Elecrron., vol. 31, pp. 1465-1473, Aug. 1995.

[5] J. Millman and H. Taub, Pulse, Digital and Switching Waveforms. New York: Mc-Graw Hill, 1965.

[6] S. M. Sze, Physics of Semiconductor Devices. New York: Wiley Eastern. pp. 783-787.

[7] L. Y. Leu, J. T. Gardner, and S. R. Forrest, "A high gain, high bandwidth $\operatorname{In}_{05} \mathrm{Ga}_{07} \mathrm{As} / \mathrm{InP}$ heterojunction phototransistor for optical communications," J. Appl. Phys., vol. 69, no. 2, Jan. 1991.

[8] Y. Wang, E. S. Yang, and W. I. Wang, "A high gain and wide dynamic range punch through heterojunction phototransistors," J. Appl. Phys., vol. 74, no. 11, Dec. 1993. 